\title{
Jacques Audiberti traduce Torquato Tasso
}

\section{Dora Bienaimé}

\section{(2) OpenEdition}

\section{Journals}

\section{Edizione digitale}

URL: http://journals.openedition.org/studifrancesi/8799

DOI: 10.4000/studifrancesi.8799

ISSN: 2421-5856

\section{Editore}

Rosenberg \& Sellier

\section{Edizione cartacea}

Data di pubblicazione: 1 octobre 2008

Paginazione: $361-370$

ISSN: 0039-2944

\section{Notizia bibliografica digitale}

Dora Bienaimé, «Jacques Audiberti traduce Torquato Tasso», Studi Francesi [Online], 155 (LII | II) |

2008, online dal 30 novembre 2015, consultato il 08 janvier 2021. URL: http://

journals.openedition.org/studifrancesi/8799; DOI: https://doi.org/10.4000/studifrancesi.8799

\section{(c) (i) (9)}

Studi Francesi è distribuita con Licenza Creative Commons Attribuzione - Non commerciale - Non opere derivate 4.0 Internazionale. 


\section{Jacques Audiberti traduce Torquato Tasso}

Audiberti pubblica nel 1946 la traduzione di alcuni Canti della Gerusalemme Liberata, (Les Flèches d'Armide, Paris, Seuil). La sua lettura appassionata del Poema del Tasso non lo esime dal porsi molteplici problemi: studia la poetica del grande autore italiano, considera i fattori storici, sociali e religiosi di quel XVI secolo così tormentato in tutta l'Europa, in particolare, in questo contesto, per la minaccia dei Turchi, giunti alle porte di Vienna per ben tre volte tra il 1529 e il 1592. Non trascura di vagliare gli scrupoli religiosi e letterari nella seconda metà del secolo, quando il Rinascimento si avvicina alla soglia del periodo barocco. Ripensa all'inquietudine scontrosa che anima il poeta: Tasso non fu un «révolté, mais à la fois conciliant, légitimiste et persécuté». S'interroga sulle motivazioni che lo indussero a narrare questa epopea, «forse per un istinto guerriero e virile, per partecipare pienamente alla vita, sia pure tramite la finzione» (J. Audiberti, Introduction, pp. 9-13).

Poi si accinge a tradurre parte dei Canti XIV, XVI e XX.

Già nel titolo Armida campeggia. La scelta del traduttore sembra essere 'istintiva', essendo «sensible au pouvoir agissant, qu'accrédite la passion ardente et mélancolique» (Introduction, p. 16). E, infatti, egli si sofferma sugli avvenimenti più favolosi del poema del Tasso, che preleva dal ricco e vasto tessuto della sanguinosa impresa della Prima Crociata (1095-1099) in cui l'epopea e l'elegia, il racconto cavalleresco e le imprese eroiche si alternano e si compendiano in un vissuto tra storia e leggenda.

Audiberti è poco sensibile agli ideali e al fanatismo religioso dei contendenti delle opposte schiere, ovviamente sono passati troppi secoli da quell'evento, ma ritiene che il Tasso, come tutti i poeti, identifichi la poesia nella storia: «la poesia è la Storia, il Canto, la Memoria» ed è convinto della «vraisemblance essentielle» con cui il poeta italiano affronta la narrazione di quegli avvenimenti, descritti con ben altra crudezza dai cronisti dell'epoca. Personalmente, egli pone in evidenza i due temi, l'amore e l'assassinio: «uomini e donne che si combattono sesso contro sesso, che ardono dal desiderio di abbracciarsi» (Introduction, p. 17). Per Audiberti, verosimile e merveilleux, si integrano nella poesia della Gerusalemme Liberata. La magia del Poema «dipenderà dal meraviglioso cristiano e sarà opera degli angeli, dei santi, demoni, maghi e incantatrici» (Introduction, pp. 9-11). Secondo il traduttore, la Crociata offriva al Tasso uno spunto valido per ingigantire personaggi di un'epopea per poi ricondurli nell'ambito del dettaglio narrativo di una azione romanzesca, non lontana dalla nostra: «Sui suoi personaggi, Tasso pose un'altra veste, l'amoureuse nudité qui revêt avec l'armure, les corps des héros, la flamme charnelle (sexuelle) qui embrase, entre autres, le couple Armide-Renaud» (Introduction, p. 13). La poesia, secondo il Tasso, annota ancora Audiberti, «può sublimare, mistificare la realtà sensibile, sulla quale ricadrà in pioggia e rugiada, una parte di questa misteriosa sublimità. Torquato Tasso ha sprigionato su di noi questa montagna adamantina di uomini, di donne, di eventi, di rime» (Introduction, pp. 13-14).

Indi, da poeta a poeta, Audiberti si accinge a tradurre, non prima di aver considerato le difficoltà, metriche, soprattutto, di una traduzione di una lingua diversa 
dalla sua. Sceglie l'esametro, un esametro da lui definito ancora una volta 'istintivo'. Osserva che l'endecasillabo della Gerusalemme Liberata, il verso classico dell'epopea italiana, «corrisponde press'a poco all'alessandrino, con minor ampiezza. Aritmeticamente sarebbe più vicino al decasillabo». Ma la poesia italiana, essendo favorevolissima alle elisioni, ne consegue che l'endecasillabo classico contiene un maggior numero di sillabe rispetto al decasillabo francese. Per tradurre in endecasillabi in un «cadre prosodique» simile a quello italiano, «sarà necessario stringere, condensare, riempire a forza. Lottare contro la tendenza classica di spiegare, rafforzare, nel tradurre, il testo da tradurre» (Introduction, pp. 18-19).

Il traduttore, che ha scritto tutte le sue poesie in rima, ritiene che in questo caso la rima sia «inopportuna», un'inutile tortura, e il più delle volte «intrasportabile» tra due lingue diverse, (Introduction, p. 22) e quindi la impiega solo occasionalmente. Ricorda i traduttori francesi della Gerusalemme Liberata, citandoli: «tutti hanno impiegato l'alessandrino rimato». La sua scelta è invece quella di scandire la narrazione poetica in esametri, in strofe di otto versi non rimati. Nel Tasso, osserva, com'è noto, che l'ottava è composta da sei versi a rima incrociata, «più due versi che rimano insieme e il cui tono, più secco, in generale smorza l'ampiezza piena e aperta della sestina» (Introduction, p. 26).

Quanto alla fedeltà al suo modello: «Je m'efforçai de calquer. Je me défendis d'ajouter à l'auteur principal et je fis de mon mieux pour que le moins possible d'omissions naquit de la rigidité de notre substance verbale au regard de l'italique malléabilité» (Introduction, p. 22). E in effetti, nella sua traduzione appaiono le costruzioni indirette, gli arcaismi possibili, le inversioni di sostantivo-aggettivo, raramente l'enjambement, tutte risorse che rispecchiano l'umile e radiosa disponibilità di fronte al Poema del Tasso.

Per le scelte tematiche Audiberti si concentra quasi esclusivamente sulla coppia Armida-Rinaldo che - circonfusa da tanti sortilegi umani e sovrumani - si orienta nell'affabulazione, si avvolge nell'aura magica che spira intorno ai due amanti, si piega alle esigenze di una realtà trasfigurata e sublimata. In virtù della poesia, Audiberti segue il Tasso nei passi in cui regnano la metamorfosi, l'incantesimo, le brusche transizioni nell'ordine della materia (L'Ile Fortunée, si disfa e si ricrea al solo gesto di Armida). Così anche nell'ordine dei sentimenti: Armida passa dall'odio all'amore, dall'amore all'odio e, infine «vinta», torna ad amare Rinaldo.

Ogni parte tradotta ha un suo titolo. In La voie des Messagers (Chant XIV), i due inviati, Ubaldo e il Danese, partiti alla ricerca di Rinaldo, seguono gli stessi passaggi magici che anche Rinaldo ha percorso, immemore e in stato di semincoscienza, catturato dai poteri di Armida, l'Incantatrice. Partiti da Ascalona, al seguito di una successione di eventi magici, giungono ai Giardini di Armida che si trovano in un'isola sperduta nell'oceano. Benché in questo reame tutto sembri immobile, almeno nelle prime ventisei ottave del Tasso, tutto ad un tratto, i giardini (Audiberti titola Les Jardins d'Armide) con un moto sinuoso e convergente, si avvolgono concentrandosi ancora nell'immobilità voluttuosa e languida dello scenario principale che rappresenta i due amanti perduti nell'estatica contemplazione della loro felicità. Come nel Poema del Tasso (a partire dalla trentaseiesima ottava), Audiberti traduce la reazione violenta di Armida contro Rinaldo, ormai determinato a seguire i Messaggeri e a far ritorno al campo cristiano (Audiberti, Chant XVI). L'ipnotica felicità di entrambi svanisce, ma non il legame di una doppia schiavitù che unisce i due amanti, tema che Audiberti riprende nel Chant XX, titolando Armide vaincue.

Con grande aderenza alla descrizione del Tasso, il traduttore segue la linea musicale del linguaggio segreto di una natura trasfigurata per effetto dei sortilegi di Armida: l'ossessione e l'incanto del labirinto in cui il tempo è sospeso. Nel Giardino, ogni 
oggetto naturale o mirabilmente artificiale, custodisce il doppio segreto di Armida, terrena e sublime nell'amore, divina e demoniaca nella futura vendetta:

Tondo è il ricco edificio; e nel chiuso grembo di lui, ch'è quasi centro al giro, Un giardin v'ha ch'adorno è sovra l'uso Di quanti più famosi unqua fioriro:

D'intorno inosservabile e confuso Ordin di logge i demon fabri ordiro; E, tra le oblique vie di quel fallace Ravvolgimento, impenetrabil giace.

(T. Tasso, Canto XVI, Prima Ottava)

Le riche édifice est rond. Au plus clos de son être, et presque au centre du tour est un jardin adorné mieux que tous les plus fameux qui jamais fleurirent. Aux bords inobservables, confus, les démons ourdirent un jeu de tunnels. Le jardin, parmi les obliques voies de l'emmêlement, gît, impénétrable.

(Audiberti, Les Jardins d'Armide)

Una voluttuosa malia spira da questi luoghi sfiorati da un alito di morte, tra brezze di cristallo e canti di uccelli, il cui virtuosismo è mirabile prodigio:

Vola tra gli altri un che le piume ha sparte

Di color vari, ed ha purpureo il rostro;

E lingua snoda in guisa larga, e parte

La voce sì, ch'assembra il sermon nostro,

Questo ivi allor continovò con arte

Tanta il parlar, che fu mirabil mostro.

Tacquero gli altri ad ascoltarlo intenti;

E fermaro $i$ sussurri in aria i venti.

(T. TAsso, Canto XVI, Ottava tredicesima)

Un oiseau vole aux plumes parsemées de couleur diverse, et pourpre le rostre. Sa langue se dénoue largement. Il parle si bien, que l'on dirait le discours nostre. Il continua d'une telle grâce à parler qu'il fut admirable monstre.

Auditeurs intenses, les autres se turent.

Les vents du ciel retinrent leurs murmures.

(Audiberti, Les Jardins d'Armide)

Quando Armida vede Rinaldo volgerle le spalle per far ritorno al campo dei Crociati, Audiberti si adegua con souplesse al lirismo ora evocativo, ora vibrante del 
Tasso, ne segue il ritmo rapido e concitato nei trasalimenti, nelle emozioni violente, nel furore di Armida. Per la sua vendetta, scrive il Tasso, «Giunta agli alberghi suoi chiamò trecento | Con lingua orrenda deità d'Averno». (Canto XVI, Ottava sessantasettesima)

E il vide (ahi fera vista!) al dolce albergo

Dar frettoloso, fuggitivo il tergo.

Volea gridar: Dove, crudel, me sola

Lasci? Ma il varco al suon chiuse il dolore

(T. TAsso, Canto XVI, Ottave 35 e 36)

Elle le vit (le voir!) au doux asile

Tourner, hâtif, le dos, comme un fuyard

(...) voulut crier: «Pourquoi

cruel, me laisses-

tu seule? (...)

(Audiberti, Les Jardins d'Armide)

Forsennata gridava: $\mathrm{O}$ tu, che porte

Teco parte di me, parte ne lassi,

O prendi l'una o rendi l'altra, o morte

Dà insieme ad ambe; arresta, arresta i passi.

(T. TAsso, Canto XVI, Ottava 40)

La forcenée hurlait: ô toi qui portes

la moitié de moi, ne me laisse l'autre!

Prends mes deux moitiées, ou rends-les ou mortes

l'une et l'autre. Arrête, arrête tes pas!

(Audiberti, Les Jardins d’ Armide) Armida:

«Beltà, sei di natura inutil dono», scrive il Tasso riferendosi alla disperazione di

Dono infelice, io ti rifiuto; e insieme

Odio l'esser reina e l'esser viva,

E l'esser nata mai: sol fa la speme

De la dolce vendetta ancor ch'io viva.

(T. TASSO, Canto XVI, ottava 66.)

Triste don, je te refuse, et, de même

Je haïs d'être reine. Je haïs d'être vive.

Je haïs d'être née, même. L'espoir blême

De me venger fait seul qu'encore je vive.

(Audiberti, Les Jardins d'Armide) 
Nel Canto XX, il tema del legame indissolubile di odio-amore è ripreso allorché Armida, con un solo gesto, cancella il mondo incantato e pensa solo alla vendetta. Ma invano prende la mira: la freccia non raggiunge l'oggetto inattaccabile per lei, il corpo di Rinaldo. Come il Tasso, Audiberti coglie l'immediatezza visiva, la tensione del gesto intenzionale di Armida: «Con occhi d'ira e di desìo tremanti», Armida prende la mira, ma la freccia che scocca non coglie il segno:

Ella stessa in su l'arco ha già lo strale.

Spingea le mani e incrudelìa lo sdegno.

Ma la placava, e n'era Amor ritegno.

$[\ldots]$

La man tre volte a saettar distese

Tre volte essa inchiodolla, e si ritenne.

Pur vinse alfin lo sdegno; e l'arco tese,

E fé volar del suo quadrel le penne.

Lo stral volò; ma con lo strale un voto,

Subito uscì, che vada il colpo a vôto.

(T. TAsso, Canto XX, ottave 62 e 63)

Armide a, sur l'arc, déjà mis la flèche.

La haïne arme ses mains, les incrimine.

Mais l'amour en elle amortit la haïne.

Toutefois sa main s'apprête à la détente.

Trois fois sa main s'incline et se retient.

L'emporte enfin la haïne. Elle tend l'arc

Et fait voler de sa flèche, les plumes.

Le dard vola, mais, avec lui, le voeu

Vola, que le coup manquât ce qu'il vise.

(Audiberti, Armide vaincue)

Audiberti, poeta, intuisce che, traducendo, «il faut jouer vaille que vaille, la scène ancienne, recommencer les gestes brûlants et scrupuleux de la découverte», affinché, con lo sguardo dell'amore e dell'ardente comunione, «la belle au bois dormant dessille ses paupières clôses depuis quatre siècles» (Introduction, p. 28). Questa è stata la sua speranza traducendo parti dei Canti XIV e XX, e più compiutamente il Canto XVI.

Agli avvenimenti precipitosi e drammatici della Gerusalemme Liberata, egli preferisce la stasi, il respiro sospeso nei luoghi trasfigurati dal potere di Armida, dal mago di Ascalona. Ne coglie, poeticamente, il fascino, lo smarrimento che essi infondono ai 'messaggeri' Crociati:

L'essors marin enfle les voiles du poème.

[...] Les navigateurs, raides dans leur suaire

de fer, entrevoient des îles, des montagnes.

\section{(Introduction à Les Jardins d'Armide)}

Così anche, si concentra sulla forza della passione sensuale che, unitamente al magico incantamento, circonfonde la coppia Rinaldo-Armida, ritrae le loro emozioni violente: ritegno, calcolo, scrupoli, disperazione soprattutto. 
E tuttavia, come ignorare, e in che modo rievocare gli episodi cruenti tra storia e leggenda della Prima Crociata? A questa necessità, Audiberti provvede a suo modo con i suoi 'résumés' in prosa. Non segue l'esattezza rapida e in certo modo impersonale dei brevi compendi ad ogni Canto che leggiamo nelle migliori edizioni della Gerusalemme Liberata. Evita la descrizione dettagliata dei fatti d'armi, tralascia le tappe del percorso di Goffredo di Buglione, le conquiste, i saccheggi, gli eccidi dei Crociati, ovvero, non tiene conto dell'afflato 'eroico' dell'epopea descritta dal Tasso che si snoda seguendo l'equilibrio costruttivo di una solenne, alta eloquenza poetica di matrice classica.

Audiberti opera una messa a fuoco concitata, ironica e benevola delle 'mirabilia', in cui può esercitare il suo estro di narratore,con una stessa libertà, uno stesso incanto:

La première croisade 's'effiloche'

Tancrède... vit une Persane, Clorinde

qui lui tira dessus et depuis pour elle,

il ard d'amour.

Renaud tue Gernaud et le charcute a coups d'épée.

Tancrède, de son coté perd son chemin.

Il se trouve soudain, prisonnier de la nuit.

Quand la nuit règne, Armide n'est pas loin.

(Audiberti, Introduction, pp. 31-33, e 35)

La successione degli avvenimenti 'eroici’ nel Poema del Tasso, doveva apparire a Audiberti, come un coacervo di azioni brutali. La carneficina lo disgusta. Non sembra condividere la tremenda osservazione del Poeta: «Bello in sì bella vista anco è l'orrore» (Canto XX, Ottava 30). La vertigine lo coglie:

Duels. Prodiges... Sanglots. Présages.

Serments. Sabbats. Tendresses. Rudesses, Guerriers

(Introduction au Chant XX)

Il suo riassunto si abbrevia, corre a folle velocità. Altrove, in un universo stregato in cui il tempo, il luogo, lo spazio sono indistinti, la rievocazione può assumere un ritmo più disteso: «La recherche devra se faire au delà des limites du monde: le fleuve s'ouvre au milieu et ils pénètrent dans les plus internes profondités de la terre, dans le sein immense de la terre», si legge nell'Introduction au Chant XIV, La Voie des Messagers). Altre volte, il suo racconto, sempre concitato, si presta a considerazioni personali sull' 'intreccio' degli avvenimenti del Poema:

Clorinde tue à tour de bras. Les flottes

cinglent. L'Histoire se fait et se disperse, songerie,

o sulla complessità del medesimo:

L'abondance des épisodes décourage le résumé.

Le résumé souffre et saigne de ne pas rapporter toutes les beautés.

(Introduction au Chant XIV) 
E ancora, nel Canto XX, la sua descrizione dei duelli, ne evidenzia soltanto la terribile istantaneità:

Altamore fend, moissonne Brunellon et Ardouin.

...

Lances brisées. Boucliers rompus.

Harnachements brisés.

(Introduction à Armide vaincue)

Gran parte degli episodi da lui tradotti hanno la loro prima origine dai versi

Qual cauta cacciatrice, Armida aspetta

Rinaldo al varco...

Su questo discrimine tra odio e amore, egli riprende il tema del Tasso sulla doppia natura umana e demoniaca di Armida, ma in lei vede gli spettacolari atti magici, il gesto che cancella il regno incantato del suo amore per trasferirlo in altri lidi al servizio dell'odio, vede in lei la donna innamorata nel gesto che tradisce, nella freccia che si blocca e non coglie il segno.

\section{Per un profilo di Jacques Audiberti}

Jean Paulhan, che è stato per Audiberti un 'nume tutelare' severo e appassionato, riteneva che nella sua vasta produzione letteraria, la poesia occupasse il primo posto.

Nato ad Antibes nel 1899, Audiberti fu poeta, romanziere, drammaturgo, saggista. Divenne noto soprattutto per le sue opere teatrali rappresentate a Parigi tra il 1941 e il $1961^{1}$.

Nei suoi romanzi, una quindicina tra il 1930 e il 1965, è riconoscibile una tendenza all'autobiografismo trasfigurato da una costante componente fantastica. Il suo linguaggio è ricco di giochi di parole, spesso soverchiato da espressioni dialettali 'antiboises' e parigine, ricco, anche, di neologismi, di accumulazioni barocche ${ }^{2}$. Jean Paulhan aveva tentato, infine con successo, di vincere la resistenza di Gaston Gallimard ad accogliere i romanzi di Audiberti, e tuttavia, anche lui esprimeva alcune riserve, gli consigliava di arginare le digressioni troppo incontrollate, sempre, e perfino nel 1964, quando Audiberti era già gravissimo:

Ton oeuvre? Je ne sais pas trop ce qu'il adviendra

de tes romans. Ils sont difficiles à cause de ces éclatements

subis vers le milieu du livre. [...]

Ah, tu auras trop dédaigné la logique, la cohérence,

l'enchaînement (et moi sans doute j'exagère leur importance) $)^{3}$.

(1) Costituiscono un insieme imponente, raccolto in Théâtre I, II, III, IV. Cito solo qualche titolo: Quoat-quoat, Le Mal court, La Fête noire, Pucelle, Altanima, La fourmi dans le corps. Sul teatro, cfr. Jeanyves Guérin, Le Théâtre d'Audiberti et le baroque.
(2) Abraxas (1938), Carnage (1942), La Poupée (1956), Les tombeaux ferment mal (1963), Monorail (1964), Dimanche m'attend (1965).

(3) Audiberti-Paulhan, Lettres (1933-1965), Les Cahiers de la N.R.F., Gallimard, 1993. Lettera datata, Lundi, (1964), p. 502. 
Numerose, anche le sue Raccolte di poesia, scritte con discontinuità: i singoli poemi spesso trascritti nelle sue lettere a Paulhan. In poesia non segue il verso libero, bensì la metrica classica in rima ${ }^{4}$.

Serpeggia, in tutta la sua opera un grande pessimismo, spesso dovuto alle condizioni materiali di una vita difficile e inquieta, assetata di affermazione e di riconoscimenti. La sua scrittura desiderante, intensa, metamorfica, pone al suo centro la donna (ma non solo), la donna come «la forme charnelle de la doctrine vivante, la puissance de la nature», così come appare anche nel suo penultimo romanzo, Monorail, nel personaggio di Hermine. Centrale è anche la Storia, in quanto memoria vivente, in particolare la Storia di una Francia imperitura, eternata nelle sue cattedrali, nelle sue antichissime vestigia.

La vita letteraria tra le due guerre sembra interessare moderatamente Audiberti. Egli ha conosciuto, e forse solo conosciuto di persona André Breton, presentatogli da Benjamin Péret. Del grande poeta, artefice del surrealismo, ha tracciato un ritratto di straordinaria intensità, tutto intriso di ammirazione e di estrema diffidenza, in una sua lettera a Jean Paulhan'. Tuttavia, una traccia del surrealismo è riconoscibile nel suo vaudeville L'Effet Glapion, mentre, nel solco della componente barocca, non prevalente nelle sue opere, potrebbe iscriversi la sua ammirazione per Torquato Tasso, nel 1946, e poi, ancora, nel 1959, il libretto lirico Altanima.

Audiberti ammirava Picasso e Dubuffet; era forse un po' lontano dagli scrittori emergenti nel secondo dopoguerra: Jean Paul Sartre, in particolare, lo impressionava per la sua quadratura e per l'intensità della sua opera.

Si è accennato al rapporto di sincera amicizia che intercorse tra Audiberti e Paulhan, malgrado la notevole differenza di età. La loro Corrispondenza ne è una prova, anche se numericamente, molto sbilanciata: poche le lettere di Jean Paulhan, disperse durante i frequenti cambiamenti di alloggio, i periodi di assenza da Parigi, nel corso dei suoi viaggi all'estero. Moltissime, invece, le sue lettere custodite nell'immenso Archivio Paulhan'.

Jean Paulhan era un uomo generoso, disponibile e sincero. Dall'alto del suo magistero e della sua indiscutibile competenza in fatto di letteratura, ha sempre aiutato anche Audiberti, non solo affidandogli la rubrica L'air du mois nella «N.R.F.», ma proponendo instancabilmente alla Casa Editrice Gallimard, i suoi romanzi, le sue raccolte di poesia.

Paulhan compare inaspettatamente al centro di alcuni Poemi di Audiberti. Uno, in particolare, scritto nel 1936, può sembrare ingenuamente laudativo. Ma, al di là del sospetto di una 'captatio benevolentiae' che farebbe torto ad entrambi gli scrittori, sorprende la giustezza del 'ritratto' e l'acuta percezione di quella persona segreta, e nello stesso tempo risoluta nelle sue scelte, che fu Jean Paulhan:

$[\ldots]$

O Jean consolateur, plus ferme que le roc,

plus tacite que l'air, plus voilé qu'un présage,

toi dont quelque détresse anime le visage

jusqu'à ce masque à bloc dont on ne revient pas

tu soie comme la pointe inerte du compas,

inerte, mais plantée, et dure dans sa fibre,

(4) Alcune sue raccolte poetiche: L'Empire et la Trappe (1929), Race des Hommes (1935), Poésies (1934-1943), Ange aux Entrailles (1964).

(5) Lettera del 19 settembre 1934 a Jean Paulhan al quale aveva chiesto di inviargli Point du Jour.

(6) Contiene tutta la Corrispondenza ricevuta dal 1904 al 1968, in parte pubblicata, in parte, e per molti anni ancora, in corso di pubblicazione. 
et fière de veiller pour sauver l'équilibre,

pour présider au noble achèvement du rond...

Jacques Audiberti è cosciente di assillare di continuo Paulhan col suo 'brouhaha' o anche «énergumat épistolaire», con il suo «purin lyrique». In tal modo definisce le sue lettere, spesso di cinque, otto pagine.

Esse compongono qualcosa di più che un diario o anche di una sequenza cronologica di idee, sentimenti, delusioni, esaltazioni giornaliere: sono un vero e proprio laboratorio delle sue opere in gestazione. Leggendole è possibile seguire l'ampio arco della sua creatività nella poesia, nel romanzo, nel teatro. In esse, Audiberti propone ritocchi essenziali, o anche minimi (una virgola, nelle sue poesie), in vista dell'opera da pubblicare - chissà quando - e, comunque, molti anni dopo. Quello che gli sta più a cuore è la consapevolezza di essere un poeta:

«Vous avez bien voulu sentir chez moi, ô mon Paulhan, la ressurrection du grand art poétique [...] le goût de la lettre en tant que matière, un sombre destin, respiratoire, la ranimée semence d'Orphée et de Gérémie» ${ }^{8}$.

Solo a partire dal luglio 1947 è possibile leggere nella Corrispondenza, alcune risposte di Jean Paulhan. Le Mal court, considerato da molti come l'opera più considerevole di Audiberti, ha avuto successo. È venuto anche il momento di proporre singoli poemi, per i «Cahiers de la Pléiade». Più frequenti sono le lettere di Paulhan nel periodo 1950-1962. Dopo molte assenze di Audiberti sempre in viaggio attraverso l'Europa, i due scrittori si incontrano in varie occasioni: le prime teatrali e quindi l'affermazione, il gioco delle bocce, gli inviti al ristorante.

Ma, già nel 1962, appaiono evidenti i sintomi gravi delle loro rispettive malattie, dovute all'età avanzata nel caso di Paulhan e, per Audiberti, un'irreparabile accorciamento della sua vita. Egli viene operato più volte. Lavora fino allo stremo, ottiene riconoscimenti. Viene insignito del Prix des critiques nel 1963, e del Grand Prix National des Lettres nel 1964, mentre Paulhan entra a far parte dell'Académie Française nello stesso anno.

Nel maggio 1965, Audiberti lascia la clinica Hartmann, dove anche Paulhan viene curato. Per poco, perché il poeta muore il 10 luglio 1965. Paulhan viene a mancare tre anni dopo, nell'ottobre 1968.

Jean Paulhan è rimasto molto colpito dalla sua malattia.

Nelle sue lettere lo incoraggiava a sperare e riceveva da lui lettere brevi, sommarie riguardo ai suoi vari ricoveri, e sempre piene di progetti.

L'11 luglio, a un giorno dalla morte di Audiberti, Paulhan mi scrive:

[...]

J'ai été peiné de la mort d'Audiberti. Il me semble qu'il était tout à fait naïvement et comme instinctivement un poète, et un grand poète. Il a horriblement souffert avant de mourir d'un cancer qui des intestins s'est porté aux reins, des reins à la prostate et de la prostate à la jambe. Mais lui, chaque fois étonné, un peu peiné, se demandant 
pourquoi on lui faisait une nouvelle opération.

Nous nous sommes trouvés quelques jours voisins

de clinique. Puis on l'a renvoyé, sans doute

jugé inguérissable. Et moi aussi - guéri, inguérissable?

On ne m’en a rien dit. Les médicins sont mystérieux.

Il 15 settembre 1965, Paulhan mi dà istruzioni per raggiungere Boissise dove ci ospita, mio marito e io, per un lungo week-end. Aggiunge:

La mort d'Audiberti m'a fait beaucoup de peine.

Aimiez-vous ses poèmes?

Nous donneriez-vous un article ( 3 à 4 pages)

pour le numéro d'hommage? Ce serait bien.

Di ritorno in Italia con gli ultimi due romanzi di Audiberti, mi trovo a corto di idee di fronte alla sua opera vastissima. Viene in mio aiuto Jean Roudaut, allora Lettore alla Scuola Normale Superiore di Pisa e all'Istituto di Lingua e Letteratura Francese dove anch'io insegnavo. Mi suggerisce l'idea brillante di considerare la traduzione francese di Audiberti, di alcuni Canti della Gerusalemme Liberata.

Il 19 ottobre 1965, Paulhan:

Chère Dora,

quelle bonne idée d'avoir songé à ce Torquato Tasso.

e, in chiusura:

Vite votre article!

Ancora, il 27 novembre,

Bien chère Dora,

Ah, mais tout devient très pressé. Je vous en prie, aussitôt que possible, votre Audiberti.

Io, nel frattempo avevo fatto il possibile per stilare cinque pagine dattiloscritte e gliele avevo inviate: troppo tardi. Del 6 dicembre è l'ultima lettera di Paulhan su questo argomento:

Bien chère Dora,

Hélas, votre étude est arrivée trop tard: le numéro

était déjà en pages, et notre imprimeur

inflexible. Mais je demande à Arland de le donner

en Janvier ou Février.

J'en aime beaucoup le ton: il y a dans votre style

on ne sait quoi d'un peu gauche, et flexible, qui

est émouvant. (le fond aussi, bien sûr. (...) ${ }^{9}$

A distanza di tanti anni ho rivisto il mio breve testo, riletto il Tasso e Les Flèches d'Armide, acquisito una conoscenza maggiore, ma pur sempre minima rispetto alla vasta opera di Audiberti, e tuttavia, tale da farmi riconsiderare il poeta nell'atto di tradurre La Gerusalemme Liberata.

DORA BIENAIMÉ

(9) Le lettere di Jean Paulhan citate e a me indirizzate, sono in parte inedite. Fanno parte di una Corrispondenza ininterrotta dal 1958 al 1967, dapprima in occasione della mia tesi di laurea sull'opera dello scrittore, in seguito sul mio lavoro di traduttrice e di saggista. 